\title{
artigo
}

\section{Avaliação da Alfa-1-Glicoproteína ácida e Mucoproteínas em pacientes com Paracoccidioidomicose tratados com Sulfametoxazol-Trimetoprim}

\author{
Evaluation of Alpha-1-Glycoprotein acid and Mucoproteins in patients with Paracoccidioidomycosis treated with \\ Sulfametoxazol-Trimetoprim
}

Evaluación del ácido Alfa-1-Glucoproteína y Mucoproteínas en pacientes con Paracoccidioidomicosis tratados con Sulfametoxazol-Trimetoprim

\section{RESUMO}

A alfa-1-glicoproteina ácida (AGP) e a mucoproteína são proteínas de fase inflamatória que aumentam suas concentrações plasmáticas quando apresentam um quadro de resposta ao estado inflamatório, representando um mecanismo de defesa do organismo. $\mathrm{O}$ objetivo do estudo foi avaliar o perfil dessas proteínas em pacientes com PCM crônica tratados com sulfametoxazol-trimetoprim (SMX-TMP) e associar os resultados encontrados com dados epidemiológicos, fatores de risco, sintomas, evolução da doença, e desfecho do tratamento. Nos métodos adotados foram analisados os prontuários de 244 pacientes com PCM crônica no período de 1998 a 2014. Destes, 134(54,92\%) pacientes fizeram exames bioquímicos das proteínas inflamatórias durante o curso da doença. Predominaram pacientes adultos de 30 a 50 anos 73(54,48\%), tabagistas 123(91,79\%), alcoólicos 60(44,78\%). Como resultado obteve-se a diminuição das proteínas inflamatórias após o tratamento ( $p=0,01803$ ). Concluindo que a AGP e a mucoproteínas são úteis como marcadores do efeito de terapia e da involução inflamatória.
\end{abstract}

DESCRITORES: Paracoccidioidomicose; Mucoproteínas; Adesão à Medicação.

\begin{abstract}
Alpha-1-acid glycoprotein (AGP) and mucoprotein are proteins of the inflammatory phase that increase their plasma concentrations when they present a response to the inflammatory state, representing a defense mechanism of the organism. The aim of the study was to evaluate the profile of these proteins in patients with chronic PCM treated with sulfamethoxazole-trimethoprim (SMX-TMP) and to associate the results found with epidemiological data, risk factors, symptoms, disease progression, and treatment outcome. In the methods adopted, the medical records of 244 patients with chronic PCM from 1998 to 2014 were analyzed. Of these, 134 (54.92\%) patients underwent biochemical tests for inflammatory proteins during the course of the disease. Adult patients aged 30 to 50 years predominated $73(54.48 \%)$, smokers $123(91.79 \%)$, alcoholics $60(44.78 \%)$. As a result, there was a decrease in inflammatory proteins after treatment ( $p=0.01803)$. In conclusion, AGP and mucoproteins are useful as markers of the effect of therapy and inflammatory involution.
\end{abstract}

DESCRIPTORS: Paracoccidioidomycosis; Mucoproteins; Medication Adherence.

\section{RESUMEN}

La glicoproteína ácida alfa-1 (AGP) y la mucoproteína son proteínas de la fase inflamatoria que aumentan sus concentraciones plasmáticas cuando presentan una respuesta al estado inflamatorio, representando un mecanismo de defensa del organismo. El objetivo del estudio fue evaluar el perfil de estas proteínas en pacientes con MCP crónica tratados con sulfametoxazol-trimetoprima (SMX-TMP) y asociar los resultados encontrados con datos epidemiológicos, factores de riesgo, síntomas, progresión de la enfermedad y resultado del tratamiento. En los métodos adoptados se analizaron las historias clínicas de 244 pacientes con MCP crónica de 1998 a 2014. De estos, 134 (54,92\%) pacientes fueron sometidos a pruebas bioquímicas de proteínas inflamatorias durante el curso de la enfermedad. Predominaron los pacientes adultos de 30 a 50 años 73 (54,48\%), fumadores 123 (91,79\%), alcohólicos $60(44,78 \%)$. Como resultado, hubo una disminución de las proteínas inflamatorias después del tratamiento ( $p=0,01803)$. En conclusión, las AGP y las mucoproteínas son útiles como marcadores del efecto de la terapia y la involución inflamatoria.

DESCRIPTORES: Paracoccidioidomicosis; Mucoproteínas; Cumplimiento de la Medicación.

RECEBIDO EM: 30/01/2021 APROVADO EM: 31/03/2021 


\section{Paula Layana Vieira Wanderley}

Especialista em Saúde da Família pela Universidade Federal do Rio Grande do Norte-UFRN (2020) Graduada em Medicina pela Faculdade de Ciências Biomédicas de Cacoal/RO-FACIMED (2018). Atualmente atua como Médica da Atenção Básica e na área da Clínica Médica.

ORCID: 0000-0002-4707-7529

\section{Carlos Eduardo Tortelli Veloso}

Especialista em Saúde da Família pela Universidade Federal do Rio Grande do Norte-UFRN (2020) Graduado em Medicina pela Faculdade de Ciências Biomédicas de Cacoal/RO-FACIMED (2018). Atualmente atua como Médico da Atenção Básica e na área da Clínica Médica.

ORCID: 0000-0003-1542-2858

\section{Tâmara Vieira Monção}

Graduanda em Medicina Universidade Federal de Roraima, UFRR ( $10^{\circ}$ semestre). ORCID: 0000-0002-7168-365X

\section{Danille Ferreira de Oliveira}

Residente do curso Ginecologia e Obstetrícia no Hospital Universitário Maria Aparecida Pedrossian da Universidade Federal do Mato Grosso do Sul (UFMS). Graduada em Medicina pela Faculdade de Ciências Biomédicas de Cacoal/RO-FACIMED (2018). Atualmente atua como Médica na área da Clínica Médica.

ORCID: 0000-0002-5357-2331

\section{INTRODUÇÃO}

A paracoccidioidomicose (PCM) é conhecida como blastomicose sul-americana, moléstia de Lutz, moléstia de Almeida ou granulomatose blastomicóide tropical. É uma micose sistêmica causada por um fungo termo-dimórfico o Paracoccidioides sp., que cresce no meio ambiente na forma de micélio, à temperatura de $25^{\circ} \mathrm{C}$, e nos tecidos à $37^{\circ} \mathrm{C}$ na forma de levedura ${ }^{1}$. É de predominância em zonas rurais do Brasil e de outros países da América do Sul, em áreas de solo fértil, levando a um impacto social e econômico². Como não é doença de notificação compulsória, desconhece-se a sua real incidência, assim como suas formas latentes ${ }^{3}$.

A PCM é de predominância em homens de meia idade ou idosos procedentes de áreas rurais brasileiras, e com reativação de foco endógeno latente, e depende de fatores relacionados tanto ao agente infeccioso quanto ao hospedeiro. Há uma menor incidência no sexo feminino ${ }^{4}$. A maioria dos casos de PCM ocorre em indivíduos cujas condições de higiene, nutricionais e socioeconômicas são precárias. Esses indivíduos costumam ser trabalhadores rurais que, por sua atividade, permanecem com mais frequência diretamente em contato com a terra e vegetais
Apresenta-se clinicamente com lesões cutâneas, na face, região perioral, mucosas orofaringolaríngea e nasal, as quais sempre se acompanham de reações ganglionares com tendência a disseminação linfática e hematogênica, acometendo órgãos internos do aparelho digestivo e respiratório ${ }^{5,6,2,7}$. A infecção pode permanecer localizada ou disseminar-se para outros órgãos por via hematogênica8. Pulmão, fígado, linfonodo, baço, rim, adrenais, mucosas e pele são os órgãos mais frequentemente afetados ${ }^{9}$.

A suspeita do diagnóstico de PCM se dá a partir de dados clínicos e epidemiológicos, mas, a confirmação se faz basicamente pela demonstração do agente etiológico em exames a fresco, cultivo e preparados histopatológicos ${ }^{10} \mathrm{e}$ o tratamento da PCM deve incluir medidas de suporte clínico e terapêutica antifúngica específica, como o uso de anfotericina $\mathrm{B}$, sulfamídicos e azólicos. Segundo Shikanai-Yasuda (2006), o paciente deve permanecer em tratamento e acompanhamento até a obtenção dos critérios de cura, com base nos parâmetros clínicos, radiológicos e sorológicos. Os pacientes deverão ser acompanhados periodicamente até apresentarem os critérios de cura ${ }^{2}$.

Os critérios de cura para paracoccidioidomicose envolvem: melhora clínica, radiológica e micológica, VHS, mucoproteínas com resultados normais, por 3 meses consecutivos ${ }^{7,11}$. A quantificação destes marcadores no soro pode ser útil na detecção do processo agudo, bem como no seu monitoramento ${ }^{12}$.

Vale ressaltar que este estudo pretende identificar o perfil de surtos inflamatórios no processo clínico de tratamento da PCM crônica cujo objetivo foi avaliar o perfil dessas proteínas em pacientes com PCM crônica tratados com sulfametoxazol-trimetoprim (SMX-TMP) e associar os resultados encontrados com dados epidemiológicos, fatores de risco, sintomas, evolução da doença, e desfecho do tratamento.

\section{MÉTODOS}

Trata-se de um estudo descritivo de análise exploratória retrospectiva de dados de prontuários de pacientes com PCM crônica que demandaram o Ambulatório de Infectologia do Hospital Universitário Júlio Muller, localizado em Cuiabá, Estado de Mato Grosso, na região sudoeste da Amazônia brasileira. A pesquisa foi realizada no ano de 2016 e teve duração de 10 meses.

A população alvo do estudo são pacientes com idade igual ou superior a 
14 anos portadores de paracoccidioidomicose atendidos no Hospital Universitário Júlio Muller no período de 1998 a 2014, do total de 244 pacientes, apenas $119(48,77 \%)$ tinham registro de mucoproteínas e 15(6,15\%) de AGP. Foram incluídos na análise apenas os pacientes com confirmação laboratorial da PCM e que estavam em primeiro tratamento da doença. Foram excluídos da análise as gestantes, crianças e pacientes que não compareceram a mais de $50 \%$ das consultas ambulatoriais previstas para o seguimento do tratamento.

A PCM foi confirmada se o paciente teve o fungo demonstrado em pelo menos um dos exames diretos de pesquisa. Foram registrados dados sobre as comorbidades tais como hanseníase, tuberculose, leishmaniose e HIV/AIDS, assim como as informações sobre os hábitos de tabagismo e etilismo, sem considerar a intensidade desses hábitos.

Os dados levantados dos prontuários foram digitados em microcomputador do pacote estatístico EpiData 3.1 (EpiData Association, Odense, Denmark) e OpenEpi. As características demográficas, epidemiológicas e clínicas foram tabuladas e adequadamente descritas em relação as alterações das proteínas alfa-1-glicoproteína ácida e das mucoproteínas na paracoccidioidomicose.

Consideraram-se alterados os exames de alfa-1-glicoproteína ácida e mucoproteínas que não estavam entre os valores de referência, de 41 a $121 \mathrm{mg} / \mathrm{dL}$ e de 2 a $4,7 \mathrm{mg} / \mathrm{dL}$, respectivamente.Os fatores associados ao desfecho do tratamento foram analisados pela razão de chance e seu intervalo de confiança de $95 \%$, quando a característica de exposição era binária. Para as variáveis independentes contínuas, a análise foi feita pela comparação das médias, por meio de teste $t$ de Student. Para todas as análises considerou-se o erro alfa de 0,05.

A pesquisa foi aprovada pelo Comitê de É tica em Pesquisa do Hospital Universitário Júlio Müller, sob o número 344.877/2013. A consulta aos prontuários foi devidamente autorizada pelo responsável clínico da instituição.
A PCM foi confirmada se o paciente teve 0 fungo demonstrado em pelo menos um dos exames diretos de pesquisa. Foram registrados dados sobre as comorbidades tais como hanseníase, tuberculose, leishmaniose e HIV/ AIDS, assim como as informações sobre os hábitos de tabagismo e etilismo, sem considerar a intensidade desses hábitos.

\section{RESULTADOS}

Foram inseridos neste estudo de análise exploratória retrospectiva os dados de prontuários dos pacientes com PCM crônica tratados com SMX-TMP do Hospital Universitário Júlio Muller localizado em Cuiabá, estado do Mato Grosso - MT, na região sudeste da Amazônia brasileira. Do total de 244 pacientes, apenas $119(48,77 \%)$ tinham registro de mucoproteínas e 15(6,15\%) de AGP.

Os pacientes que fizeram os exames de proteínas inflamatórias foram distribuídos de acordo com as características demográficas, epidemiológicas, clínicas e bioquímicas. Quanto ao sexo foram 134 pacientes, sendo $132(98,51 \%)$ homens e apenas $2(1,49 \%)$ mulheres. O grupo foi dividido em três faixas etárias: 14-30 anos; 31-50 anos; > 50 anos. Maior alteração das proteínas inflamatórias ocorreu na faixa etária de 31-50anos, 36(26,87\%). Quanto à profissão, observamos que em um universo de 106 pacientes, 5(4,13\%) eram agricultores, $8(6,61 \%)$ pedreiros, 56(46,28\%) lavrador, 2(1,65\%) garimpeiros, $50(41,32 \%)$ outros, a maioria das profissões relacionadas com manejo do solo.

Quanto a procedência, 117(87,97\%) eram de MT, 14(10,53\%) de Rondônia, e 2(1,50\%) de outros estados. Analisando os fatores agravantes, $123(91,79 \%)$ eram tabagistas, 60(44,78\%) eram alcoolistas, $32(23,88 \%)$ apresentavam diversas comorbidades e 3(2,24\%) tinham coinfecção PCM-HIV/AIDS. Observando os sintomas, os mais frequentes foram: emagrecimento $93(69,40 \%)$, febre $35(26,12 \%)$, tosse $91(67,91 \%)$, escarro $59(44,03 \%)$, linfadenomegalia 66(49,25\%), gânglios $34(25,37 \%)$, odinofagia 56(41,79\%), rouquidão $34(25,37 \%)$, astenia $50(40 \%)$, dispneia 59(43,70\%). Avaliando a adesão ao tratamento, 91(73,39\%) aderiram. A proporção das alterações bioquímicas foram: AGP 11(73,33\%), e mucoproteínas 54(45,38\%) (Tabela 1).

$\mathrm{Na}$ análise entre os fatores associados e o aumento das proteínas inflamatórias observa-se que dos 132 homens analisados 65(49,24\%) tiveram alterações. Não se 
Tabela 1: Características demográficas, epidemiológicas e clínicas de adultos com PCM crônica em adultos tratados com SMX-TMP, Cuiabá - MT, 2016.

\begin{tabular}{|c|c|c|c|}
\hline CARACTERÍSTICA & CATEGORIA & $\mathbf{N}$ & $\%$ \\
\hline \multirow[t]{2}{*}{ Sexo } & Masculino & 132 & $98,51 \%$ \\
\hline & Feminino & 2 & $1,49 \%$ \\
\hline \multirow[t]{3}{*}{ Etária } & $1(14 \mid-30)$ & 2 & $1,49 \%$ \\
\hline & $2(30 \mid-50)$ & 73 & $54,48 \%$ \\
\hline & $3(\geq 50)$ & & \\
\hline 59 & $44,03 \%$ & & \\
\hline Profissão & Agricultor & 5 & $4,13 \%$ \\
\hline \multirow[t]{4}{*}{$n=106$} & Pedreiro & 8 & $6,61 \%$ \\
\hline & Lavrador & 56 & $46,28 \%$ \\
\hline & Garimpeiro & 2 & $1,65 \%$ \\
\hline & Outros & 50 & $41,32 \%$ \\
\hline UF & MT & 117 & $87,97 \%$ \\
\hline \multirow[t]{2}{*}{$n=133$} & $\mathrm{RO}$ & 14 & $10,53 \%$ \\
\hline & Outros & 2 & $1,50 \%$ \\
\hline \multirow[t]{4}{*}{ Fatores agravantes } & Tabagismo & 123 & $91,79 \%$ \\
\hline & Alcoolismo & 60 & $44,78 \%$ \\
\hline & Comorbidades & 32 & $23,88 \%$ \\
\hline & HIV/AIDS & 3 & $2,24 \%$ \\
\hline \multirow[t]{10}{*}{ Sintomas } & Emagrecimento & 93 & $69,40 \%$ \\
\hline & Febre & 35 & $26,12 \%$ \\
\hline & Tosse & 91 & $67,91 \%$ \\
\hline & Escarro & 59 & $44,03 \%$ \\
\hline & Linfadenomegalia & 66 & $49,25 \%$ \\
\hline & Gânglios & 34 & $25,37 \%$ \\
\hline & Odinofagia & 56 & $41,79 \%$ \\
\hline & Rouquidão & 34 & $25,37 \%$ \\
\hline & Astenia & 50 & $40,00 \%$ \\
\hline & Dispneia & 59 & $43,70 \%$ \\
\hline \multicolumn{2}{|c|}{ Adesão referida ao tratamento } & 91 & $73,39 \%$ \\
\hline \multirow[t]{2}{*}{ Alterações bioquímicas } & Alfa-1-Glicoproteína acida & 11 & $73,33 \%$ \\
\hline & Mucoproteína & 54 & $45,38 \%$ \\
\hline
\end{tabular}

FONTE: Hospital Universitário Júlio Muller, Cuiabá -MT, 2016. Obs: a variação do n deve-se a ausência de informações nos prontuários. observou tendência estatisticamente significante na comparação das faixas etárias com as alterações nas proteínas de fase agu$\mathrm{da}$ (Tabela 2). Tanto os pacientes tabagistas quanto os alcoolistas demonstraram respectivamente um aumento estatisticamente significante das proteínas de fase aguda, $(\mathrm{p}=0,03568)$ e $(\mathrm{p}=0,01653)$ (Tabela 2).

Quando se compara a presença dos sintomas com as alterações das proteínas inflamatórias verificam-se: emagrecimento $54(58,06 \%)$, febre $20(57,14 \%)$, tosse 49 (53,85\%), escarro $39(66,10 \%)$, linfadenomegalia 34 (51,52\%), gânglios 17 $(50,00 \%)$, odinofagia $29(51,79 \%)$, rouquidão 19 (55,88\%), astenia $24(48,00 \%)$, dispneia 30 (50,85\%). Observou-se aumento das proteínas de fase aguda em pacientes que apresentaram escarro, com relevância estatisticamente significante $(\mathrm{p}=0,000301)$ (Tabela 3$)$.

A análise entre as alterações das mucoproteínas e o desfecho clínico na PCM crônica em adultos tratados com SMX-TMP demonstrou que 36 (48\%) dos pacientes que tiveram alterações das proteínas de fase aguda evoluíram com cura clínica, 13 (68,42\%) apresentaram sequelas e 1 paciente evoluiu com óbito, sem associação estatisticamente significante (Tabela 4).

Verificou-se diminuição estatisticamente significante das proteínas de fase aguda em pacientes com PCM crônica na fase pós-tratamento quando comparada à fase pré-tratamento ( $\mathrm{p}=0,01803)$ (Tabela 5).

\section{DISCUSSÃO}

O estudo do perfil da AGP e das mucoproteínas em pacientes com PCM crônica contribui de forma importante para o acompanhamento clínico mais detalha-

Tabela 2: Análise da associação das alterações das proteínas inflamatórias e dos fatores de risco em adultos com PCM crônica em adultos tratados com SMX-TMP, Cuiabá - MT, 2016.

\begin{tabular}{|c|c|c|c|c|c|c|c|c|c|}
\hline \multirow[t]{2}{*}{ FATOR } & \multirow[t]{2}{*}{ CATEGORIA } & \multicolumn{4}{|c|}{$\begin{array}{l}\text { ALTERAÇÃO DE MUCOPROTEÍNAS E ALFA-1- } \\
\text { GLICOPROTEÍNA ÁCIDA }\end{array}$} & & \multirow[t]{2}{*}{ OR } & \multirow[t]{2}{*}{ IC95\% } & \multirow[t]{2}{*}{ P } \\
\hline & & SIM & $(\%)$ & NÃO & $(\%)$ & TOTAL & & & \\
\hline \multirow[t]{2}{*}{ Sexo } & Masculino & 65 & $49,24 \%$ & 67 & $50,76 \%$ & 132 & - & - & $0,5265^{*}$ \\
\hline & Feminino & 0 & $0,00 \%$ & 2 & $100,00 \%$ & 2 & - & - & - \\
\hline
\end{tabular}




\section{artigo}

Wanderley, P.L.V.; Veloso, C.E.T.; Monção, T.V.; Oliveira, D.F.

Avaliação da Alfa-1-Glicoproteína ácida e Mucoproteínas em pacientes com Paracoccidioidomicose tratados com Sulfametoxazol-Trimetoprim

\begin{tabular}{|c|c|c|c|c|c|c|c|c|c|}
\hline \multirow[t]{3}{*}{ Etária } & $1(14 \mid-30)$ & 1 & $50,00 \%$ & 1 & $50,00 \%$ & 2 & - & - & - \\
\hline & $2(30 \mid-50)$ & 36 & $49,32 \%$ & 37 & $50,68 \%$ & 73 & - & - & - \\
\hline & $3(\geq 50)$ & 28 & $47,46 \%$ & 31 & $52,54 \%$ & 59 & - & - & - \\
\hline Adesão & Não & 12 & $36,36 \%$ & 21 & $63,64 \%$ & 33 & & & \\
\hline Tabagismo & $\operatorname{Sim}$ & 63 & $51,22 \%$ & 60 & $48,78 \%$ & 123 & 4,677 & $1,062-32,89^{* *}$ & 0,03568 \\
\hline \multirow[t]{2}{*}{ Alcoolismo } & Sim & 36 & $60,00 \%$ & 24 & $40,00 \%$ & 60 & 2,313 & $1,097-4,957$ & 0,01653 \\
\hline & Não & 29 & $39,19 \%$ & 45 & $60,81 \%$ & 74 & & & \\
\hline \multirow[t]{2}{*}{ HIV } & $\operatorname{Sim}$ & 1 & $33,33 \%$ & 2 & $66,67 \%$ & 3 & 0,526 & $0,008738-10,33$ & $>0,999^{*}$ \\
\hline & Não & 64 & $48,85 \%$ & 67 & $51,15 \%$ & 131 & & & \\
\hline \multirow[t]{2}{*}{ Tuberculose } & $\operatorname{Sim}$ & 6 & $50,00 \%$ & 6 & $50,00 \%$ & 12 & 0,850 & $0,2139-3,373$ & $>0,999^{*}$ \\
\hline & Não & 66 & $54,10 \%$ & 56 & $45,90 \%$ & 122 & & & \\
\hline \multirow[t]{2}{*}{ Leishmaniose } & $\operatorname{Sim}$ & 4 & $44,44 \%$ & 5 & $55,56 \%$ & 9 & 0,840 & $0,159-4,107$ & $>0,999^{*}$ \\
\hline & Não & 61 & $48,80 \%$ & 64 & $51,20 \%$ & 125 & & & \\
\hline \multirow[t]{2}{*}{ Comorbidade } & Sim & 21 & $65,63 \%$ & 11 & $34,38 \%$ & 32 & 1,148 & $0,5505-2,401$ & 0,6883 \\
\hline & Não & 44 & $43,14 \%$ & 58 & $56,86 \%$ & 102 & & & \\
\hline
\end{tabular}

Tabela 3: Análise da associação das manifestações clínicas e dos fatores de risco em adultos com PCM crônica em adultos tratados com SMX-TMP, Cuiabá - MT, 2016.

\begin{tabular}{|c|c|c|c|c|c|c|c|c|c|}
\hline \multirow[t]{2}{*}{ FATOR } & \multirow[t]{2}{*}{ CATEGORIA } & \multicolumn{4}{|c|}{$\begin{array}{c}\text { ALTERAÇÃO DE MUCOPROTEÍNAS E ALFA- } \\
\text { 1-GLICOPROTEÍNA ÁCIDA }\end{array}$} & \multirow[b]{2}{*}{ TOTAL } & \multirow[t]{2}{*}{ OR } & \multirow[t]{2}{*}{ IC95\% } & \multirow[t]{2}{*}{$\mathbf{P}$} \\
\hline & & SIM & $(\%)$ & NÃO & $(\%)$ & & & & \\
\hline \multirow[t]{2}{*}{ Emagrecimento } & Sim & 54 & $58,06 \%$ & 39 & $41,94 \%$ & 93 & 0,645 & $0,2703-1,482$ & 0,2638 \\
\hline & Não & 28 & $68,29 \%$ & 13 & $31,71 \%$ & 41 & & & \\
\hline \multirow[t]{2}{*}{ Febre } & Sim & 20 & $57,14 \%$ & 15 & $42,86 \%$ & 35 & 1,660 & $0,7142-3,93$ & 0,1964 \\
\hline & Não & 44 & $44,44 \%$ & 55 & $55,56 \%$ & 99 & & & \\
\hline \multirow[t]{2}{*}{ Tosse } & $\operatorname{Sim}$ & 49 & $53,85 \%$ & 42 & $46,15 \%$ & 91 & 1,959 & $0,8817-4,461$ & 0,07205 \\
\hline & Não & 16 & $37,21 \%$ & 27 & $62,79 \%$ & 43 & & & \\
\hline \multirow[t]{2}{*}{ Escarro } & Sim & 39 & $66,10 \%$ & 20 & $33,90 \%$ & 59 & 3,637 & $1,69-8,047$ & 0,000301 \\
\hline & Não & 26 & $34,67 \%$ & 49 & $65,33 \%$ & 75 & & & \\
\hline \multirow[t]{2}{*}{ Linfadenomegalia } & Sim & 34 & $51,52 \%$ & 32 & $48,48 \%$ & 66 & 1,266 & $0,6089-2,643$ & 0,4925 \\
\hline & Não & 31 & $45,59 \%$ & 37 & $54,41 \%$ & 68 & & & \\
\hline \multirow[t]{2}{*}{ Gânglios } & $\operatorname{Sim}$ & 17 & $50,00 \%$ & 17 & $50,00 \%$ & 34 & 1,083 & $0,4614-2,542$ & 0,8402 \\
\hline & Não & 48 & $48,00 \%$ & 52 & $52,00 \%$ & 100 & & & \\
\hline \multirow[t]{2}{*}{ Odinofagia } & Sim & 29 & $51,79 \%$ & 27 & $48,21 \%$ & 56 & 1,251 & $0,5953-2,639$ & 0,52 \\
\hline & Não & 36 & $46,15 \%$ & 42 & $53,85 \%$ & 78 & & & \\
\hline \multirow[t]{2}{*}{ Rouquidão } & Sim & 19 & $55,88 \%$ & 15 & $44,12 \%$ & 4 & 1,483 & $0,633-3,526$ & 0,3192 \\
\hline & Não & 46 & $46,00 \%$ & 54 & $54,00 \%$ & 100 & & & \\
\hline Astenia & Sim & 24 & $48,00 \%$ & 26 & $52,00 \%$ & 50 & 1,112 & $0,5103-2,424$ & 0,7696 \\
\hline
\end{tabular}




\begin{tabular}{|c|c|c|c|c|c|c|c|c|c|}
\hline & Não & 34 & $45,33 \%$ & 41 & $54,67 \%$ & 75 & & & \\
\hline \multirow[t]{2}{*}{ Dispneia } & $\operatorname{Sim}$ & 30 & $50,85 \%$ & 29 & $49,15 \%$ & 59 & 1,483 & $0,633-3,526$ & 0,3192 \\
\hline & Não & 36 & $47,37 \%$ & 40 & $52,63 \%$ & 76 & & & \\
\hline
\end{tabular}

FONTE: Hospital Universitário Júlio Muller, Cuiabá -MT, 2016. Quiquadrado linear

Tabela 4: Análise da associação das alterações das proteínas inflamatórias e do desfecho clínico em adultos com PCM crônica, Cuiabá - MT, 2016.

\begin{tabular}{|c|c|c|c|c|c|c|c|c|c|}
\hline \multirow[t]{2}{*}{ FATOR } & \multirow[t]{2}{*}{ CATEGORIA } & \multicolumn{4}{|c|}{$\begin{array}{l}\text { ALTERAÇÃO DE MUCOPROTEÍNAS E ALFA- } \\
\text { 1-GLICOPROTEÍNA ÁCIDA }\end{array}$} & \multirow[b]{2}{*}{ TOTAL } & \multirow[t]{2}{*}{ OR } & \multirow[t]{2}{*}{ IC95\% } & \multirow[t]{2}{*}{$\mathbf{P}$} \\
\hline & & SIM & $(\%)$ & NÃO & $(\%)$ & & & & \\
\hline Cura clínica & Não & 12 & $36,36 \%$ & 21 & $63,64 \%$ & 33 & & & \\
\hline Sequela & $\operatorname{Sim}$ & 13 & $68,42 \%$ & 6 & $31,58 \%$ & 19 & 2,451 & $0,6384-10,33$ & 0,1368 \\
\hline \multirow[t]{2}{*}{ Óbito } & $\operatorname{Sim}$ & 1 & $33,33 \%$ & 2 & $66,67 \%$ & 3 & 0,348 & $0,005546-7,186$ & 0,3749 \\
\hline & Não & 25 & $59,52 \%$ & 17 & $40,48 \%$ & 42 & & & \\
\hline
\end{tabular}

Tabela 5: Análise da associação das alterações das proteínas inflamatórias nas fases pré e pós-tratamento com SMXTMP na PCM crônica, Cuiabá - MT, 2016.

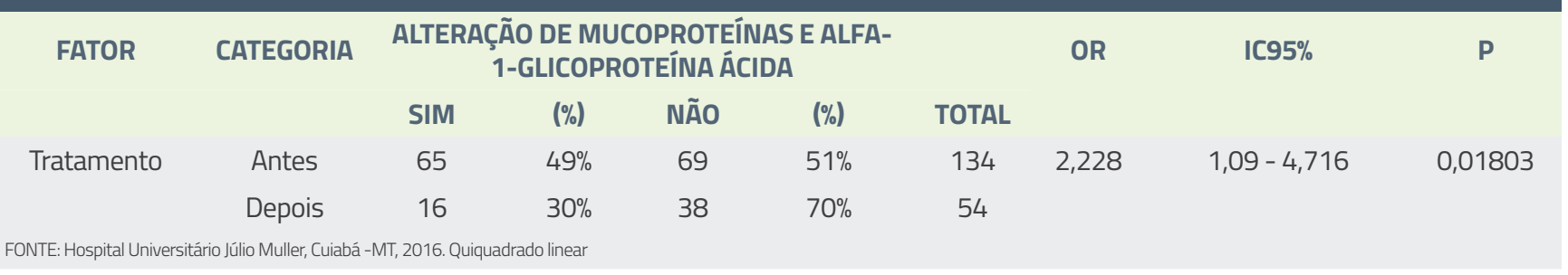

do e a avaliação de resposta terapêutica adequada. Poucas são as publicações que cercam o tema e raros são os estudos que abordam o seguimento das proteínas inflamatórias após o início do tratamento ${ }^{13}$. Por isso, esse estudo vem avaliar os prontuários de 134 pacientes que realizaram os exames de proteínas inflamatórias durante o seguimento da doença.

Houve predomínio dos pacientes das regiões endêmicas, que vive em área rural ou exerceu atividade agrícola relacionadas ao manejo do solo nas primeiras décadas de vida, tendo possivelmente adquirido a infecção nessa época, embora as manifestações clínicas surjam anos depois, tendo características de doença ocupacional. Apresentando assim, um importante fator de correlação epidemiológica, e de relevância sob o ponto de vista de saúde pública por acometer indivíduos em sua fase de plena atividade produtiva ${ }^{14,2,10}$.

O envolvimento do sistema linfático é uma das manifestações clínicas mais comuns da PCM, correspondendo a 66(49,25\%) casos e apresentando frequência semelhante à de outras séries de casos na literatura. $\mathrm{O}$ comprometimento pulmonar esteve presente em até $90 \%$ dos pacientes, e em até $25 \%$ dos pacientes é o único órgão envolvido, sendo a dispneia um dos sintomas mais observados nos pacientes com PCM ${ }^{15,10}$.

O presente estudo está de acordo com autores supracitados, demonstrando que após o tratamento da PCM crônica com SMP-TMP, houve queda substancial das proteínas de fase aguda, o que sugere que AGP e mucoproteínas são úteis como marcadores laboratoriais da eficácia da terapia e da involução inflamatória. 


\section{artigo}

Wanderley, P.L.V.; Veloso, C.E.T.; Monção, T.V.; Oliveira, D.F.

Avaliação da Alfa-1-Glicoproteína ácida e Mucoproteínas em pacientes com Paracoccidioidomicose tratados com Sulfametoxazol-Trimetoprim

Considerando a magnitude da PCM no Brasil, e a relevância das alterações laboratoriais encontradas, sugere-se a necessidade da recomendação desses marcadores inflamatórios durante o seguimento do tratamento da PCM crônica como útil marcador laboratorial de resposta ao tratamento e de regressão inflamatória, pode ser considerado também como um dos critérios de cura da PCM crônica.

\section{CONCLUSÃO}

A diminuição estatisticamente significante das proteínas de fase aguda em paciente com PCM crônica na fase pós-tratamento, quando comparada à fase pré-tratamento pode sugerir a recomendação da solicitação desses marcadores inflamatórios durante o seguimento clínico de pacientes com PCM crônica, especialmente para indicar boa resposta ao tratamento e de regressão da atividade inflamatória.
Na PCM crônica, pacientes alcoolistas

e tabagistas

apresentaram

aumento

significativo

das proteínas

inflamatórias
Na PCM crônica, pacientes alcoolistas e tabagistas apresentaram aumento significativo das proteínas inflamatórias, o que sugere que essas elevações séricas podem demonstrar tendência de que esse estilo de vida influencia diretamente no aumento das proteínas inflamatórias.

$\mathrm{O}$ aumento estatisticamente significativo das proteínas de fase aguda em pacientes que apresentaram escarro como manifestação clínica corrobora com o grande componente inflamatório deflagrado por esta micose.

O conhecimento sobre a PCM é de grande interesse médico-epidemiológico. Porém há fatores que dificultam os estudos, como a doença não ser de notificação compulsória na maioria do país. Esse estudo vem ressaltar a necessidade de novas pesquisas para avaliar o papel das proteínas inflamatórias nas doenças negligenciadas.

\section{REFERÊNCIAS}

1. Capelari, Marcos Maurício; Detoni, Ângelo Antonio; Marzola, Clóvis; Toledo-Filho, João Lopes. Paracoccidioidomicose: apresentação de caso clínico com o uso de fluconazol e miconazol. Revista Eletrônica de Odontologia da Academia Tiradentes de Odontologia, Bauru, v. fe 2009, n. 2, p. 173-189, 2009.

2. Shikanai-Yasuda, M. A. "Paracoccidioidomycosis Treatment." Revista do Instituto de Medicina Tropical de São Paulo 57, p. 3137, 2015.

3. Muniz, Maria Angélica Soares et al. Paracoccidioidomicose pulmonar: aspectos na tomografia computadorizada de alta resolução. Radiol Bras, São Paulo, v. 35, n. 3, p. 147-154, June 2002.

4. PALMEIRO, M et al. Paracoccidioidomicose-Revisão da Literatura. Scientia Medica 15(4):274-278. 2005.

5. Paniago, A. M. M, Aguiar J. I. A, Aguiar E. S, Cunha R. V, et al. Paracoccidioidomicose: estudo clínico e epidemiológico de 422 casos observados no Estado do Mato Grosso do Sul. Rev Soc Bras Med Trop. 2003; 36: 455-459.

6. Verli, Flaviana Dornela et al. Perfil clínico-epidemiológico dos pacientes portadores de paracoccidioidomicose no Serviço de Estomatologia do Hospital São Lucas da Pontifícia Universidade Católica do Rio Grande do Sul. Rev. Soc. Bras. Med. Trop., Uberaba, v. 38, n. 3, p. 234-237, May 2005.

7. Wanke, B. and M. A. Aidê. "Capítulo 6 - Paracoccidioidomicose." Jornal Brasileiro de Pneumologia 35, p. 1245-1249, 2009.

8. Nucci, M. Treatment of paracoccidioidomycosis. Em: UpToDate, Post TW (Ed), UpToDate, Waltham, MA. (Acessado em 02 de novembro de 2016.)

9. Franco, M.; montenegro, M.R.G. Anatomia patológica. In Paracoccidiodomicose: Blastomicose Sulamericana. Editado por G. del Negro, C. da S. Lacaz, A. M. Fiorillo. São Paulo, Sarvier, 97117, 1982.

10. Peçanha, P. M. Aspectos epidemiológicos e clínicos da Paracoccidioidomicose no Estado do Espírito Santo (dissertação). Vitória: Universidade Federal do Espírito Santo. 2012.

11. Ferreira, B. M. et al. Validação do reagente alfa-1glicoproteina acida turbidimétrico. Monografia (Aperfeiçoamento/Especialização em Biotecnologia) - Universidade Federal de Lavras, 2006.

12. Picheth, G., et al. "Mucoproteína versus alfa-1-glicoproteína ácida: o que quantificar?" Jornal Brasileiro de Patologia e Medicina Laboratorial 38, p. 87-91, 2002.

13. Levorato, A. D. Avaliação da bioquímica sanguínea de pacientes com paracoccidioidomicose, tratados com cotrimoxazol ou itraconazol. (Dissertação) Botucatu: Universidade Estadual Paulista "Júlio De Mesquista Filho" UNESP. 2014.

14. Fornajeiro, Nair et al. Inquérito epidemiológico sobre a paracoccidioidomicose utilizando a gp43 em dois municípios do noroeste do Paraná, Brasil. Rev. Soc. Bras. Med. Trop., Uberaba, v. 38, n. 2, p. 191-193, Apr. 2005.

15. Costa, Marlos Augusto Bitencourt et al. Manifestações extrapulmonares da paracoccidioidomicose. Radiol Bras, São Paulo, v. 38, n. 1, p. 45-52, Feb. 2005. 